\title{
Electronic Stopping Power in LiF from First Principles
}

\author{
J. M. Pruneda, ${ }^{1,2}$ D. Sánchez-Portal, ${ }^{3,4}$ A. Arnau, ${ }^{3,5,6}$ J. I. Juaristi, ${ }^{3,5,6}$ and Emilio Artacho ${ }^{3,7}$ \\ ${ }^{1}$ Instituto de Ciencia de Materiales de Barcelona (ICMAB-CSIC) Campus de Bellaterra, 08193 Barcelona, Spain \\ ${ }^{2}$ Department of Physics, University of California, Berkeley, California 94720, USA \\ ${ }^{3}$ Donostia International Physics Center, Paseo Manuel de Lardizabal 4, 20018 San Sebastian, Spain \\ ${ }^{4}$ Centro de Física de Materiales, Centro Mixto CSIC-UPV/EHU, Apartado 1072, 20080 San Sebastian, Spain \\ ${ }^{5}$ Centro Mixto CSIC-UPV/EHU, Facultad de Química, Apartado 1072, 20080 San Sebastian, Spain \\ ${ }^{6}$ Departamento de Física de Materiales, Facultad de Química, Apartado 1072, 20080 San Sebastian, Spain \\ ${ }^{7}$ Department of Earth Sciences, Downing Street, University of Cambridge, Cambridge CB2 3EQ, United Kingdom*
}

(Received 12 June 2007; published 3 December 2007)

\begin{abstract}
Using time-dependent density-functional theory we calculate from first principles the rate of energy transfer from a moving proton or antiproton to the electrons of an insulating material, LiF. The behavior of the electronic stopping power versus projectile velocity displays an effective threshold velocity of $\sim 0.2$ a.u. for the proton, consistent with recent experimental observations, and also for the antiproton. The calculated proton/antiproton stopping-power ratio is $\sim 2.4$ at velocities slightly above the threshold ( $v \sim 0.4$ a.u. ), as compared to the experimental value of 2.1. The projectile energy loss mechanism is observed to be extremely local.
\end{abstract}

DOI: 10.1103/PhysRevLett.99.235501

The interaction of charged particles with matter has been a subject of extensive research since the discovery of subatomic particles [1]. Ions moving through solids gradually transmit their kinetic energy to electronic excitations of the host and deposit it along their path. The maximum of this deposited energy per unit path length is the so-called Bragg peak and it occurs shortly before the particle stops. Hence the importance of studying the electronic energy loss of slow ions (with velocities below the Bohr velocity) traveling through solids. For metals, the understanding of this problem has been steadily progressing over the years [1]. For insulators, however, experimental results remain unexplained even for simple systems. This is particularly true at low velocities (the threshold effect) $[2,3]$, where the contribution from nuclear collisions conceals the electronic stopping [4].

A detailed quantitative knowledge of these processes is required to understand the damage produced in materials when exposed to radiation. For ceramic materials devised for the encapsulation of nuclear waste [5] the prediction of durability over extremely long times is crucial. Radiationdamage simulations performed to date [6,7] rely on empirical force fields obtained from fits to low-energy properties. The actual interatomic forces could be enormously altered, however, by the local electron heating produced by the electronic stopping.

In the semiclassical formalism, the electronic energy loss rate is given by the response of the system to the external potential, $\frac{d E}{d t}=-Z \mathbf{v} \cdot \mathbf{E}^{\text {ind }}$, where $Z$ and $\mathbf{v}$ are the charge and velocity of the projectile, and $\mathbf{E}^{\text {ind }}$ is the induced electric field in the target material. Hence, to first order, linear-response theory can be used to give the stopping power (SP) - the energy lost per unit path length - in terms of the dynamical dielectric function of the material. For metals, this formalism shows that the SP is linear at low
PACS numbers: 61.85.+p, 34.50.Bw, 61.80.Jh, 61.82.Ms

velocities, $\frac{d E}{d x} \sim(Z e)^{2} v[8,9]$, reflecting that no minimum energy is required to excite electron-hole pairs. It must be remembered, however, that the dielectric approach is not valid at low ion velocities, and nonlinear effects cannot be neglected [1].

A different behavior is expected in wide band-gap insulators, given the finite energy required to excite electrons [10]. For protons moving through noble gases [11], which also display a large minimum excitation energy, a velocity threshold has been observed experimentally [12], and explained in terms of the quantization of energy levels and charge exchanges [13]. However, for solid insulators the situation is unclear. No threshold effects were originally observed in $\mathrm{Al}_{2} \mathrm{O}_{3}, \mathrm{SiO}_{2}$, or $\mathrm{LiF}$, and the linear dependence $d E / d x \propto v$ was observed down to velocities of about 0.3 a.u. $[2,14,15]$.

For protons under grazing incidence in $\operatorname{LiF}(001)$, and below $\sim 0.2$ a.u. a threshold behavior was reported [16]. Under these conditions the proton does not penetrate the solid, and charge exchange is identified as the dominant mechanism for electronic stopping, with local electron capture from $\mathrm{F}^{-}$ions, giving rise to $\mathrm{H}^{0}$ and $\mathrm{H}^{-}$. More recent experiments on thin $\mathrm{LiF}$ films show an apparent velocity threshold near 0.1 a.u. [3]. The different experimental setup (transmission) suggests a different physical stopping mechanism, based on electron-hole pair excitations. A threshold behavior is expected, and has been qualitatively predicted from linear response and from perturbation theory calculations. These approximations, however, grossly underestimate the SP at low velocities, thus exaggerating the threshold [17]. A theoretical description beyond these approximations is needed.

In this Letter we present a first-principles approach to the nonperturbative study of realistic solid-ion interactions. We follow the explicit time evolution of the electronic 
states of the host crystal as an external particle propagates through the system, by means of time-dependent densityfunctional theory (TD-DFT) [18]. We use here this scheme to understand the SP threshold effect and stopping mechanisms in LiF, a well-studied characteristic insulator, finding reasonable agreements with measured data. Most importantly, however, this study sets the scene for a promising line of theoretical simulations, assesses its possibilities and offers new insights into the stopping process.

The energy transmitted to the electrons from a constantvelocity moving ion is monitored. Total energy is thus not conserved since the energy loss of the projectile is neglected (its large mass ensures a negligible decline in its velocity on the time scales of the simulations). As the charged particle moves, the time-dependent Kohn-Sham (KS) equation [18] defines the dynamics of effective single-particle states (and thereby the electronic density and energy) under the external potential generated by the projectile and the crystal of $\mathrm{Li}$ and $\mathrm{F}$ nuclei.

The calculations were done using the SIESTA $a b$ initio method [19,20], in its time-evolving TD-DFT implementation [21], using the instantaneous local density approximation (LDA) to exchange and correlation [22]. The $1 s$ core electron pair of $\mathrm{F}$ was replaced by a norm-conserving pseudopotential in the fully nonlocal form. The $1 \mathrm{~s}$ electrons of Li were explicitly included in the calculation. A double- $\zeta$ polarized basis was considered for the valence electrons (single- $\zeta$ for the $1 s$ of Li), with an energy shift of $100 \mathrm{meV}$ [20]. The grid cutoff [20] for integration was $118 \mathrm{Ry}$. The lattice parameter obtained for bulk $\mathrm{LiF}$ was $3.98 \AA$, slightly smaller than the experimental value $4.03 \AA$, as expected for LDA. The projectile (proton, $p$, or antiproton, $\bar{p}$ ) was described by the bare $\pm \frac{1}{r}$ potential [23] instead of pseudopotentials, in order to treat $p$ and $\bar{p}$ on the same footing.

Periodic boundary conditions were used throughout. The supercell size was chosen so as to minimize the spurious effects of the repetition while keeping manageable computational demands. After convergence tests, a $4 \times 4 \times 4$ supercell of 128 atoms was selected, such that a particle moves $\sim 11 \AA$ before reentering the box. A single $k$ point $(\Gamma)$ was used for integrations in the Brillouin zone. The projectile was initially put in the center of a crystal cage and the time-independent DFT solution was obtained to define the initial state for the subsequent evolution. It was then moved with constant velocity in a straight line trajectory along a [110] channel. A rigid lattice was assumed [4]. The time-dependent KS equation was then solved numerically by discretizing time as described in Ref. [21]. Using a time step of $1 \mathrm{asec}$, the wave functions were then propagated for several femtoseconds. The total electronic energy was recorded as a function of time and the SP, $\frac{d E}{d x}$, was extracted.

Figure 1 shows the total electronic energy of the system as a function of displacement for different velocities of the projectile. At low velocities the adiabatic behavior is re-

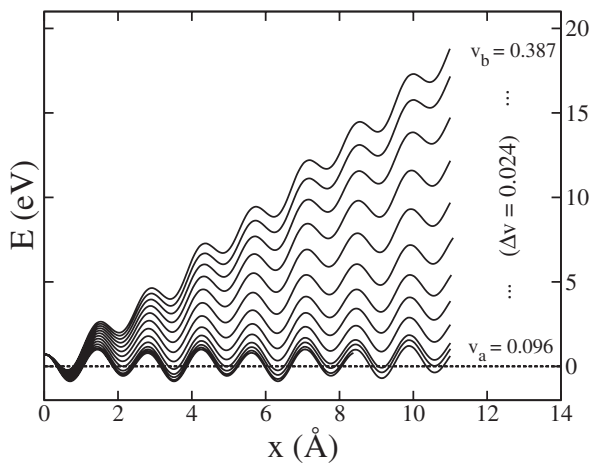

FIG. 1. Total electronic energy as a function of displacement for several proton velocities.

covered, with no net energy transfer, just the expected oscillation of the total energy with the position of the projectile in the crystal. At higher velocities the oscillations are superimposed to an underlying energy increase with time. After a remarkably short transient period (around $0.3 \mathrm{fs}$ ), the energy increase stabilizes to an essentially stationary regime, in which the energy difference between consecutive equivalent lattice positions of the projectile remains constant. The SP is then extracted from the average slope of this stationary regime. The results for $p$ and $\bar{p}$ are presented in Fig. 2 (closed and empty circles, respectively) along with the available experimental data. A velocity threshold is apparent in our calculated SP and the measurements for $p$ [3]. This is in contrast with the linear behavior observed for metals [1]. The calculated thresholds of $\sim 0.2$ a.u. are consistent with proton experiments, $[3,15]$ much smaller than those predicted for insulators by linear-response theory [17], and a forecast for antiproton experiments at lower energies.

A considerable discrepancy is still observed when comparing the calculated and the measured values. A factor of $\sim 2$ would be accounted for by the relation between channelling conditions and the average over random trajectories $[24,25]$. Its effect is shown in Fig. 2. Technical reasons account for part of the remaining discrepancy [26]. For $p$ we can improve the basis by including orbitals along the projectile's path (a $s p$ single- $\zeta$ hydrogen basis set every $0.5 \AA$ ). This increases the SP (crosses in Fig. 2), e.g., from 1.6 to $2.8 \mathrm{eV} / \AA$ for $v=0.46$ a.u., and brings it into better agreement with experiment. Unfortunately, such an augmented basis set is only justified for $p$. At a more fundamental level, discrepancies are expected from the errors in electronic spectra around the band gap given by instantaneous LDA in TD-DFT (even if it includes a RPA correction on KS eigenvalues) [27].

Figure 2 shows a clear difference between $p$ and $\bar{p}$ (we henceforth focus on the results for the unbiased basis [26]), in contrast with the invariance under charge-sign change expected from perturbative treatments. Experiments show that the energy loss for protons is approximately twice as 


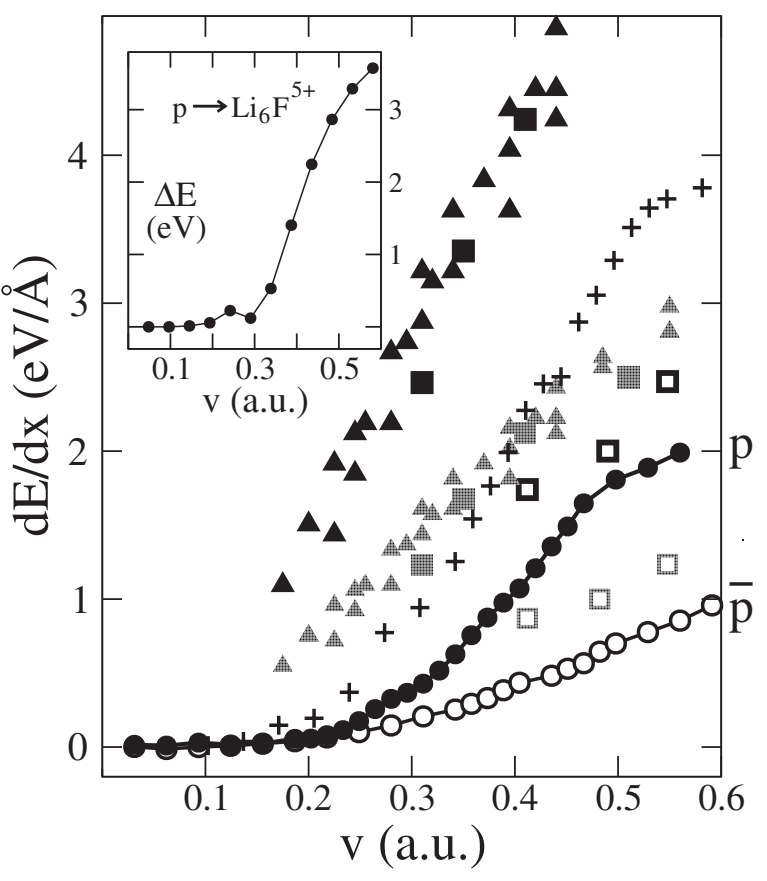

FIG. 2. Electronic stopping power $\frac{d E}{d x}$ as a function of particle velocity $v$ for $p$ (full symbols) and $\bar{p}$ (empty symbols). Circles are the calculations while triangles and squares indicate, respectively, the measured values of Refs. [3,15]. Gray triangles and squares are these measured values scaled by $1 / 2$, for direct comparison with the calculations, which only considered channelling trajectories. Crosses are our results for $p$ with additional basis orbitals along the projectile's path (see text). The inset shows the excess energy acquired by a octahedral $\mathrm{Li}_{6} \mathrm{~F}^{5+}$ isolated cluster on the passage of a $p$ on an analogous trajectory to that of the solid.

high as for antiprotons [15], a consequence of the different kind of screening [28]. Slightly above the threshold $(v \sim$ 0.4 a.u.) we obtain a SP ratio of 2.4 , that compares well with the experimental value of 2.1 [15].

Linear response predicts a quadratic dependence of the $\mathrm{SP}$ on the projectile charge $q$. Figure 3 presents the calculated SP over $q^{2}$. The smooth behavior at the origin indicates that there are no substantial biases in the way the stopping mechanism is described for $p$ and $\bar{p}$. The slope near the origin corresponds to the $q^{3}$ dependence, while the bending corresponds to $q^{4}$ and higher terms. The displayed

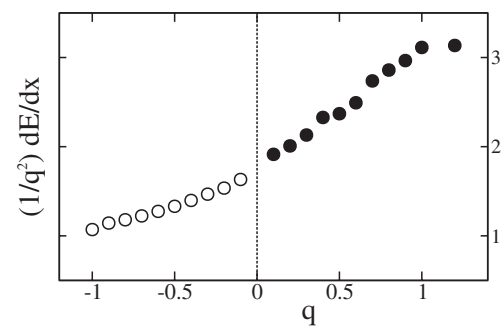

FIG. 3. Dependence of $\frac{1}{q^{2}} \frac{d E}{d x}$ with the charge $q$ of the external projectile, for $v=0.46$ a.u.. The stopping power is in $\mathrm{eV} / \AA$, and $q$ in electron charge units. behavior, and particularly the sign of both terms, is similar to that observed for metals [29].

We now analyze the locality of the energy absorption in terms of Mulliken charges [30]. Although they are known to be unreliable in their explicit dependence on the basis set, their changes for the fixed basis set are much more indicative. By comparing the atomic charges obtained dynamically and adiabatically (a static self-consistent calculation with the external potential at the same instantaneous position), insights are obtained into the stopping process. Figure 4 shows this comparison for the proton case, where the following is observed: (i) The screening of the proton is enhanced in the dynamic case (our basis set describes this screening by a charge transfer from $\mathrm{F}$ to $\mathrm{Li}$ ); (ii) a delay is apparent in the dynamical screening, which is the origin of the dissipation; (iii) the electronic excitation process is extremely local: the changes observed in atoms closest to the trajectory are much larger than any other, and the dynamic effect is only noticeable when the projectile is very close to the ion.

The locality of the energy transfer is confirmed when calculating the energy absorbed by a small cluster of $\mathrm{LiF}$ $\left(\mathrm{Li}_{6} \mathrm{~F}^{5+}\right)$. The inset of Fig. 2 shows a striking similarity in the overall $v$-dependence of the energy absorbed by the cluster and the SP in the solid. If we took the effective path length in the cluster as $1.4 \AA$ (the number of valence electrons in the cluster corresponds to one formula unit), both SPs would be indeed very similar [31]. In the solid, the energy accumulated along the path would then diffuse away at longer time scales (corresponding to the effective band width), defining a wake. A tentative definition of local energy [32] shows well differentiated time scales for the excitation by the projectile, and for the ensuing outdiffusion (not shown). The short-ranged initial excitation can be rationalized in terms of the electronic localization length scale relevant to dielectric response [33], which is expected to be very short for LiF. Similar locality in the energy loss mechanism has been recently found in jellium clusters, however [34].

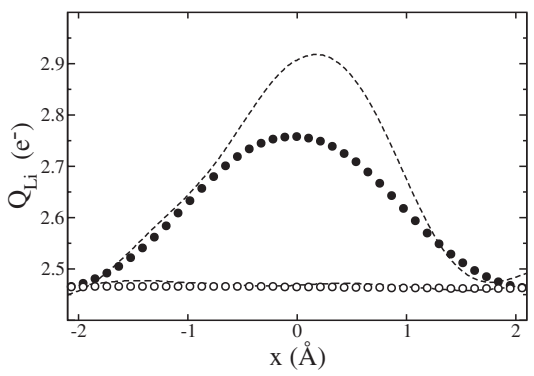

FIG. 4. Electron population on Li atoms close to the proton trajectory versus position of the projectile along it. Adiabatic (dynamic) charges are shown by circles (dashed lines). The position of the maximum in the adiabatic curve occurs when the projectile is closest to the atom $(\sim 1.2 \AA)$. The almost constant line corresponds to a second closest $\mathrm{Li}$ atom, $2.3 \AA$ away from the trajectory. 
A characterization of the charge state of the projectile $[35,36]$ has not been attempted. The present calculations allow for the establishment of charge states of any kind within the constraint that the electronic charge of the simulation box is conserved. It is clear that the midgap state travels with the proton, and that it becomes partly populated. It is not clear, however, that the charge associated to that state represents a meaningful definition of the charge state of the projectile, since whether the screening charge builds up as it passes or travels with it is not determined by the population of that state. This effect will be explored in further works.

In conclusion, we have presented a general approach for the nonperturbative first-principles study of the electronic stopping power in solids. New insights into the electronic SP in insulators have been provided for protons and antiprotons in $\mathrm{LiF}$.

We thank P. M. Echenique, E. Salje, and I. Nagy for interesting discussions. E. A. acknowledges the hospitality of the Donostia International Physics Centre. This work has been funded by the EC's Marie Curie program, UK's BNFL and NERC, the Spanish MEC, the UPV/EHU and the Basque Government through the Etortek program.

*Permanent address.

[1] P. M. Echenique, F. Flores, and R. H. Ritchie, in Solid State Physics: Advances in Research and Applications, edited by H. Ehrenreich and D. Turnbull (Academic, New York, 1990), Vol. 43, p. 229.

[2] K. Eder, D. Semrad, P. Bauer, R. Golser, P. Maier-Komor, F. Aumayr, M. Peñalba, A. Arnau, J. M. Ugalde, and P. M. Echenique, Phys. Rev. Lett. 79, 4112 (1997).

[3] M. Draxler, S. P. Chenakin, S. N. Markin, and P. Bauer, Phys. Rev. Lett. 95, 113201 (2005).

[4] A. Mertens and H. Winter, Phys. Rev. Lett. 85, 2825 (2000).

[5] I. Farnan, H. Cho, and W. J. Weber, Nature (London) 445, 190 (2007).

[6] K. Trachenko, M. T. Dove, E. Artacho, I. T. Todorov, and W. Smith, Phys. Rev. B 73, 174207 (2006).

[7] B. P. Uberuaga, R. Smith, A. R. Cleave, F. Montalenti, G. Henkelman, R. W. Grimes, A. F. Voter, and K. E. Sickafus, Phys. Rev. Lett. 92, 115505 (2004).

[8] R. H. Ritchie, Phys. Rev. 114, 644 (1959).

[9] M. Kitagawa and Y.H. Ohtsuki, Phys. Rev. B 9, 4719 (1974).

[10] E. Artacho, J. Phys. Condens. Matter 19, 275211 (2007).

[11] R. Cabrera-Trujillo, J. R. Sabin, Y. Öhrn, and E. Deumens, Phys. Rev. Lett. 84, 5300 (2000).

[12] R. Golser and D. Semrad, Phys. Rev. Lett. 66, 1831 (1991).

[13] P.L. Grande and G. Schiwietz, Phys. Rev. A 47, 1119 (1993).

[14] J. I. Juaristi, C. Auth, H. Winter, A. Arnau, K. Eder, D. Semrad, F. Aumayr, P. Bauer, and P. M. Echenique, Phys. Rev. Lett. 84, 2124 (2000).
[15] S.P. Møller, A. Csete, T. Ichioka, H. Knudsen, U. I. Uggerhøj, and H.H. Andersen, Phys. Rev. Lett. 93, 042502 (2004).

[16] C. Auth, A. Mertens, H. Winter, and A. Borisov, Phys. Rev. Lett. 81, 4831 (1998).

[17] M. Peñalba, J. I. Juaristi, E. Zarate, A. Arnau, and P. Bauer, Phys. Rev. A 64, 012902 (2001).

[18] E. Runge and E. K. U. Gross, Phys. Rev. Lett. 52, 997 (1984).

[19] P. Ordejón, E. Artacho, and J. M. Soler, Phys. Rev. B 53, R10 441 (1996).

[20] J. M. Soler, E. Artacho, J. D. Gale, A. García, J. Junquera, P. Ordejón, and D. Sánchez-Portal, J. Phys. Condens. Matter 14, 2745 (2002).

[21] A. Tsolakidis, D. Sánchez-Portal, and R. M. Martin, Phys. Rev. B 66, 235416 (2002).

[22] J. P. Perdew and A. Zunger, Phys. Rev. B 23, 5048 (1981).

[23] The electrostatic energy divergence due to the charge of the projectile in periodic boundary conditions is avoided by using $\frac{e^{-r / \rho}}{r}$ instead of $\frac{1}{r}$ and converging the SP with respect to $\rho$. This is equivalent to a compensating homogeneous background. The $\frac{1}{r}$ cusp has been removed using a Gaussian charge smoothening of a width dictated by the grid cutoff used in SIESTA [20].

[24] P. L. Grande and G. Schiwietz, Phys. Lett. A 163, 439 (1992).

[25] J. J. Dorado and F. Flores, Phys. Rev. A 47, 3062 (1993).

[26] The basis set used throughout this work has been the one defined by atomic orbitals of target atoms, so that no explicit bias was introduced in the comparison of $p$ and $\bar{p}$. There is a finite-basis saturation effect at high velocities, but that is observed at higher velocities than the ones studied here. Although the absolute value of the SP is not completely converged by our basis set, the convergence for both the relation proton/antiproton and the respective thresholds is satisfactory.

[27] H. Appel, E. K. U. Gross, and K. Burke, Phys. Rev. Lett. 90, 043005 (2003).

[28] R. Vincent, J. I. Juaristi, and I. Nagy, Phys. Rev. A 71, 062902 (2005).

[29] P. M. Echenique, A. Arnau, M. Peñalba, and I. Nagy, Nucl. Instrum. Methods Phys. Res., Sect. B 56-57, 345 (1991).

[30] R. S. Mulliken, J. Chem. Phys. 23, 1833 (1955).

[31] TD-DFT is known to provide better results for small systems [27], we thus expect the spectra of our cluster to be more accurate than that of bulk.

[32] The energy associated to a basis function $|\mu\rangle$ is defined as $E_{\mu}=\sum_{\nu} \rho_{\mu \nu} H_{\nu \mu}$, where $\rho_{\mu, \nu}$ and $H_{\mu \nu}$ are the density and Hamiltonian matrices, respectively. Note that it does not include double-counting terms.

[33] I. Souza, T. Wilkens, and R. M. Martin, Phys. Rev. B 62, 1666 (2000).

[34] M. Quijada, A. G. Borisov, I. Nagy, R. Díez-Muiño, and P. M. Echenique, Phys. Rev. A 75, 042902 (2007).

[35] A. Närmann, R. Monreal, P. M. Echenique, F. Flores, W. Heiland, and S. Schubert, Phys. Rev. Lett. 64, 1601 (1990).

[36] T. Prokscha, E. Morenzoni, D. G. Eshchenko, N. Garifianov, H. Glückler, R. Khasanov, H. Luetkens, and A. Suter, Phys. Rev. Lett. 98, 227401 (2007). 